\title{
MAPK pathways and alphaB-crystallin phosphorylation in brain: a focus on aging and Alzheimer's disease
}

\author{
Natalia Muraleva \\ Molecular mechanisms of aging \\ Institute of Cytology and Genetics SB RAS \\ Novosibirsk, Russia \\ Myraleva@bionet.nsc.ru
}

\begin{abstract}
Accumulation of intracellular damage and protein aggregates is an universal hallmark of aging and accompanies the development of some age-related diseases include Alzheimer's disease (AD). Alpha-B-Crystallin (CryaB) as the molecular chaperone contributes maintenance of proteostasis by prevention of aggregation of proteins (e.g. amyloid beta) and enables their correct refolding. CryaB activity is regulated by MAPK signaling pathway (MAPKsp) through its phosphorylation. Nevertheless, the link between changes in MAPK-dependent CryaB phosphorylation with age and the development of $A D$ remains unclear. Here, we examined p38 MAPK- and ERK-dependent phosphorylation of CryaB in the brain of Wistar rats with normal aging and senescence-accelerated OXYS rats at the different stages of the development of AD-like pathology, including the presymptomatic stage. The most significant changes identified in the p38 MAPK-dependent CryaB phosphorylation. The level of pSer59-CryaB in the brain of Wistar rats increased with the age on the background of p38-MAPKsp activation. Similar but more significant changes accompanied the development of AD-like pathology in OXYS rats. The activation of ERK1/2-dependent CryaB phosphorylation (pSer45-CryaB) was detected at the early age and at the late stages of AD-like pathology in OXYS, while changes in the ERK1/2 signaling pathway were detected in Wistar rats with age. Thus, alteration of MAPK-dependent phosphorylation CryaB occurs with the normal aging. Manifestation and progression of the signs of the AD occurs against the background of activation of p38MAPK-dependent phosphorylation of CryaB. Activation of EPK-dependent CryaB phosphorylation is characteristic of the preclinical and progressive stage of the AD-like pathology.
\end{abstract}

Keywords - aging, phosphorylation, MAPK pathways, alphaBcrystallin, Alzheimer's disease, OXYS rats

\section{Introduction}

Accumulation of intracellular damage and protein aggregates is an universal hallmark of aging. Maintenance of protein proteostasis is attained through precisely coordinated systems that must rapidly correct unwanted proteomic changes. The points of cross talk between the unfolded protein response and MAPK signaling pathways that may contribute to our understanding of the mechanisms of alteration of proteostasis processes with aging and with development of age-related diseases including Alzheimer's disease (AD). MAPK signaling network regulates cell survival and death responses following a variety of stresses including misfolded protein response stress. Two subfamilies of MAPKs (p38MAPK and ERK1/2) participate in this by regulating the activity of the alphaBcrystallin (CryaB) through its phosphorylation. CryaB as a molecular chaperone prevents aggregation of proteins (e.g. amyloid beta) and enables their correct refolding. Nevertheless, the link between changes in MAPK-dependent CryaB phosphorylation with the normal aging and the development of AD remains unclear. Here, we examined CryaB, phospho- (p-)
Ser59-CryaB and p-Ser45-CryaB protein amounts in the brain of Wistar rats with normal aging and senescence-accelerated OXYS rats at different stages of the development of AD-like pathology. We compared this result with the changes in expression of genes involved in the p38 MAPK and ERK 1/2 signaling pathways and the content of key proteins of these pathways in brain of Wistar and OXYS rats.

\section{Materials and Methods}

The work was carried out on male OXYS rats and Wistar rats (control) aged 20 days, 5, and 18 months based in the Center of Genetic Resources of Laboratory Animals at the Institute of Cytology and Genetics, Siberian Branch of the Russian Academy of Sciences (Novosibirsk, Russia).

To detect changes in the expression of genes involved in MAPK- and ERK1/2 signaling pathways in Wistar and OXYS rats, we analyzed RNA-Seq data obtained previously [1].

Standard techniques of western blott analysis and immunohistochemistry were used to examine of content of CryaB, p-Ser45-CryaB, p-Ser59-CryaB and key proteins of MAPK- and ERK1/2 signaling in the prefrontal cortex and hippocampus of Wistar and OXYS rats. The data were subjected to two-way analysis of variance in the Statistica 8.0 software.

\section{Results}

Here, we showed that in the prefrontal cortex of rats OXYS and Wistar p-Ser59-CryaB was detected only in the detergentinsoluble fraction. Its level did not differ between OXYS and Wistar rats at the age of 20 days. Increase of p-Ser59-CryaB content occurred with age in rats of both strains. The manifestation and progression of signs of AD in OXYS rats were accompanied by an increase in the level of p-Ser59-CryaB relative to Wistar rats in the prefrontal cortex and hippocampus. It should be noted that the content of p-Ser59-CryaB in the detergent-insoluble fraction was significantly higher in OXYS rats, which indicates the formation of stronger links of p-Ser59CryaB with the target molecule. Indeed, immunohistochemical staining of brain sections revealed in the brain of 18-month-old OXYS rats the joint localization of p-Ser59-CryaB with toxic beta amyloid (A $\beta 1-42)$. CryAB phosphorylation (S59) is mediated through activation of $\mathrm{p} 38$ protein kinase. A significant increase in the p38 MAPK protein content in the detergentinsoluble fraction was found in the cortex of OXYS rats aged 5 and 18 months, while its level did not change in Wistar rats. In these age groups, OXYS rats showed an increase in the level of phosphorylation of $\mathrm{p} 38$ MAPK protein in both protein fractions. A similar study of the contents of p38MAPK and p-p38MAPK in the hippocampus of OXYS and Wistar rats during the same age periods revealed similar results with the level of these proteins in the prefrontal cortex. 
According to the analysis of transcriptomes of the prefrontal cortex and hippocampus, the development of AD signs in OXYS rats is accompanied by an increase in the number of differentially expressed genes (DEGs) involved in the p38 MAPK signaling pathway. Changes in the mRNA level of genes in OXYS rats are associated with such categories of gene ontologies as the cascade of protein kinases, phosphorylation, calcium signaling, and the protein kinase activating signaling pathway.

Next, we showed that in the prefrontal cortex and hippocampus of OXYS and Wistar rats p-Ser45-CryaB was contained in detergent-soluble and detergent-insoluble protein fractions. The level of p-Ser45-CryaB in OXYS rats was higher than in the control Wistar rats at the age of 20 days and 18 months. It should be noted that the accumulation of p-Ser45CryaB in the detergent-insoluble fraction increased already at the preclinical stage of AD-like pathology in OXYS rats. CryaB phosphorylation (S45) is mediated through activation of the ERK1/2 signaling pathway controlled by ERK1/2 protein kinases. A significant increase in the protein content of ERK1/2 was found in the prefrontal cortex and hippocampus of OXYS rats at all studied ages. According to the analysis of transcriptomes of the prefrontal cortex and hippocampus, the progression of signs of AD in OXYS rats was accompanied by an increase in the number of DEG involved in the ERK1 / 2 signaling pathway.

\section{CONCLUSION}

Thus, alteration of MAPK-dependent phosphorylation of CryaB is happening with the normal aging. Activation of EPK1/2-dependent CryaB phosphorylation occurs with age and is characteristic for the preclinical and progressive stages of the AD-like pathology. Manifestation and progression of the signs of the $\mathrm{AD}$ occur against the background of activation of p38MAPK-dependent phosphorylation of CryaB and changes in gene expression of this signaling pathway. An increase in the level of p-Ser59-CryaB and its joint localization with $A \beta 1-42$ can be considered as a response to the accumulation of toxic protein aggregates in the brain, which is an important part of the endogenous mechanism of $\mathrm{AD}$ development.

\section{ACKNOWLEDGMENT}

Microscopy was performed at the Microscopy Center of the Institute of Cytology and Genetics, SB RAS, Russia. This work was supported by grant from the RFBR (\#18-015-00336).

\section{REFERENCES}

[1] Stefanova, N.A., Maksimova, K.Y., Rudnitskaya, E.A., Muraleva, N.A., Kolosova, N.G. Association of cerebrovascular dysfunction with the development of Alzheimer's disease-like pathology in OXYS rats. BMC Genomics. 2018, 9, 19(Suppl 3):75. https://doi.org/10.1186/s12864-0184480-9. 